\title{
Neuronal Coherence as a Mechanism of Effective Corticospinal Interaction
}

Jan-Mathijs Schoffelen et al.

Science 308, 111 (2005);

DOI: $10.1126 /$ science.1107027

This copy is for your personal, non-commercial use only.

If you wish to distribute this article to others, you can order high-quality copies for your

colleagues, clients, or customers by clicking here.

Permission to republish or repurpose articles or portions of articles can be obtained by following the guidelines here.

The following resources related to this article are available online at www.sciencemag.org (this information is current as of November 13, 2012 ):

Updated information and services, including high-resolution figures, can be found in the online version of this article at:

http://www.sciencemag.org/content/308/5718/111.full.html

Supporting Online Material can be found at:

http://www.sciencemag.org/content/suppl/2005/03/29/308.5718.111.DC1.html

This article cites 26 articles, 13 of which can be accessed free:

http://www.sciencemag.org/content/308/5718/111.full.html\#ref-list-1

This article has been cited by 97 article(s) on the ISI Web of Science

This article has been cited by 60 articles hosted by HighWire Press; see:

http://www.sciencemag.org/content/308/5718/111.full.html\#related-urls

This article appears in the following subject collections:

Neuroscience

http://www.sciencemag.org/cgi/collection/neuroscience 
we found an elevated rate of recombination in the chimpanzee.

20. www.hapmap.org/.

21. S. B. Gabriel et al., Science 296, 2225 (2002).

22. I. Ebersberger, D. Metzler, C. Schwarz, S. Paabo, Am. J. Hum. Genet. 70, 1490 (2002).

23. H. Watanabe et al., Nature 429, 382 (2004).

24. D. Falush, M. Stephens, J. K. Pritchard, Genetics 164 1567 (2003).

25. S. R. Myers, R. C. Griffiths, Genetics 163, 375 (2003)

26. M. K. Halushka et al., Nat. Genet. 22, 239 (1999).
27. We thank the members of the Altshuler, Daly, and Donnelly laboratories and the participants in the International Haplotype Map Project for helpful discussions. D.A. is a Charles E. Culpeper Scholar of the Rockefeller Brothers Fund and a Burroughs Wellcome Fund clinical scholar in translational research. P.D. is supported by $\mathrm{NIH}$, Wellcome Trust, Nuffield Trust, the SNP Consortium, and Wolfson Foundation. G.A.T.M. is supported by $\mathrm{NIH}$, The Royal Society, and the SNP Consortium. D.R. is the recipient of a Career Development Award in Biomedical Science from the Burroughs Wellcome Fund.
Supporting Online Material

www.sciencemag.org/cgi/content/full/1105322/DC1

Materials and Methods

Tables S1 and S2

Figs. $\mathrm{S} 1$ to $\mathrm{S} 5$

References and Notes

16 September 2004; accepted 1 February 2005 Published online 10 February 2005 .

10.1126/science 1105322

Include this information when citing this paper.

\title{
Neuronal Coherence as a Mechanism of Effective Corticospinal Interaction
}

\begin{abstract}
Jan-Mathijs Schoffelen, ${ }^{1,2 *}$ Robert Oostenveld, ${ }^{1,3}$ Pascal Fries ${ }^{1,2}$
Neuronal groups can interact with each other even if they are widely separated. One group might modulate its firing rate or its internal oscillatory synchronization to influence another group. We propose that coherence between two neuronal groups is a mechanism of efficient interaction, because it renders mutual input optimally timed and thereby maximally effective. Modulations of subjects' readiness to respond in a simple reaction-time task were closely correlated with the strength of gamma-band ( 40 to 70 hertz) coherence between motor cortex and spinal cord neurons. This coherence may contribute to an effective corticospinal interaction and shortened reaction times.
\end{abstract}

Within the central nervous system, the main occupation of a given group of neurons is to interact with other groups. It is commonly assumed that the influence of one neuronal group on another is primarily determined by the mean rate of action potentials generated (1). However, recent evidence suggests that a postsynaptic neuron actively compensates for slow changes in mean input rate and primarily responds to precisely synchronous input barrages (2-5). Studies in awake and behaving animals suggest that neuronal groups increase their impact on target groups through precise oscillatory synchronization $(6,7)$, and it has been hypothesized that this might constitute a general mechanism for regulating the flow of information in the nervous system (8).

Here, we propose a mechanism that might greatly amplify the effects of oscillatory synchronization. Activated groups of neurons typically oscillate in the beta or gamma frequency bands $(9,10)$ and thereby undergo temporally predictable excitability fluctuations. Synaptic input to an oscillating neuronal target group will be maximally effective if it arrives within a few milliseconds of the excitability peaks of the target (11). Thus, for a neuronal

${ }^{1}$ F. C. Donders Centre for Cognitive Neuroimaging, Radboud University Nijmegen, 6525 EK Nijmegen, Netherlands. ${ }^{2}$ Department of Biophysics, Radboud University Nijmegen, 6525 EZ Nijmegen, Netherlands. ${ }^{3}$ Center for Sensory-Motor Interaction (SMI), Aalborg University, Fredrik Bajers Vej 7 D-3, 9220 Aalborg, Denmark.

*To whom correspondence should be addressed. E-mail: jan.schoffelen@fcdonders.ru.nl indirectly measure the activity of the corresponding spinal neuronal group. Activity in the corresponding left motor cortex was assessed with magnetoencephalography (MEG). Corticospinal coherence (coherence between motor cortex and spinal cord) could then be assessed with standard analysis methods (16).

We investigated the effect of a manipulation of corticospinal interaction on corticospinal coherence. We used the well-studied behavioral effect that in simple reaction-time tasks, the hazard rate of the go-cue (17) determines the subjects' readiness to respond as operationalized by shortened reaction times $(18,19)$. Subjects extended their right wrist to elevate their hand against the lever of a force meter to bring the measured force into a specified window. After a baseline period, a visual stimulus appeared and subjects had to keep the wrist extension until the stimulus changed speed at an unpredictable moment in time (fig. S1). The crucial experimental manipulation was to systematically modulate the hazard rate of the stimulus' speed change. In the UP-schedule, a stimulus change became more and more likely the longer the stimulus was on without change. In the DOWN-schedule, a stimulus change became less and less likely. A given subject was trained in three sessions on one of the schedules before neuronal activity was recorded in a fourth session. After several days' break, the same was done for the other sched- group to provide maximally effective input, it should be coherent with the target group.

We tested whether this potential mechanism has a functional role in human cognition and whether this role can be demonstrated through a behavioral correlate of coherence between distant groups of neurons. We studied coherence between motor cortex and spinal alpha-motoneurons (12-15) (Fig. 1). Because a spinal motoneuron and the corresponding muscle fibers form a motor unit with one-toone correspondence of their action potentials, we used the electromyogram (EMG) of the right musculus extensor carpi radialis longus to
Fig. 1. (A and B) Readiness to respond induces corticospinal gamma-band coherence. In (A), corticospinal coherence [z-transformed (30), averaged over the six sensors highlighted in (C) and (D)] is shown before (blue) and after (red) onset of the visual stimulus; (B) shows the difference. Horizontal bars in (A) indicate frequency ranges used in (C) (blue bar) and (D) (red bar). Horizontal bar in (B) indicates frequency band with significant difference $(P<0.05$, nonparametric randomization test). (C) Topography of beta-band coherence before the onset of the visual stimulus. (D) Topography of gamma-

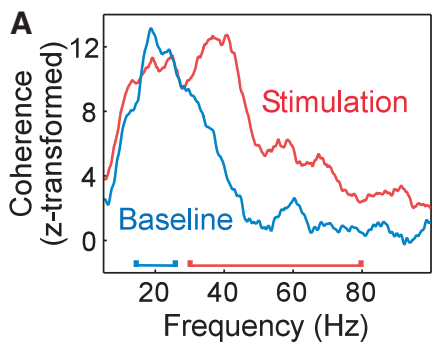

C

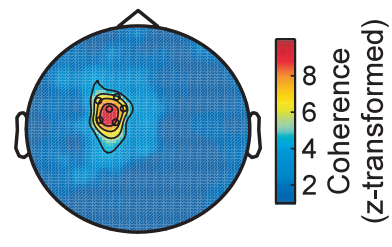

B

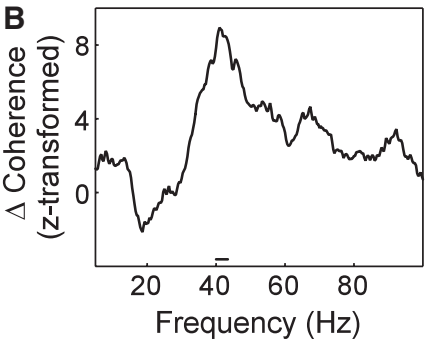

D

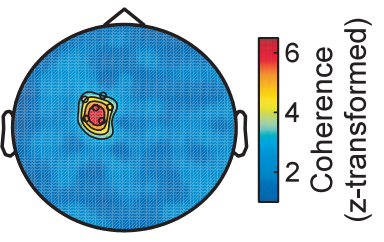

band coherence after the onset of the visual stimulus. 


\section{R E P O R T S}

Fig. 2. Effect of increasing hazard rate on reaction times, cortical power, and corticospinal coherence $(n=6$ subjects). (A) Hazard rate (gray line) with the resulting reaction times (pink line). Note the inverted reaction time axis at the right. (B) Timefrequency representation of MEG power over contralateral motor cortex. (C) Time courses of the cortical power in the gamma band (40 to $70 \mathrm{~Hz}$ ) (red line) and the beta band (15 to $30 \mathrm{~Hz}$ ) (blue line). For comparison of power time courses and hazard rate, two scaled copies of the latter are shown (gray lines), one upright (gamma power) and one inverted (beta power). (D) Pearson correlation coefficient between hazard rate and cortical power as a function of frequency of the latter. Bars indicate significant frequency bands $(P<0.05$, nonparametric randomization test, corrected for multiple comparisons). (E) Time-frequency representation of corticospinal coherence. (F) Time course of corticospinal coherence in the gamma band (40 to $70 \mathrm{~Hz}$ ). As in (C), the gray line is a scaled copy of the hazard rate. (G) Pearson correlation coefficient between hazard rate and corticospinal coherence as a function of frequency of the latter. Bars indicate significant frequency bands $(P<0.05$, nonparametric randomization test, corrected for multiple comparisons).

ule. The sequence of schedules was counterbalanced across the six subjects studied (20).

The behavioral results from the recording sessions confirm earlier findings (Figs. 2A and $3 \mathrm{~A})$. When the hazard rate increased linearly in the UP-schedule, reaction times decreased in close correspondence (Pearson correlation coefficient $r=-0.93, P<0.0001$, randomization test); the reverse was found for the DOWN-schedule $(r=-0.95, P<$ 0.0001 ). Thus, subjects had implicitly learned the hazard rate for the two schedules and were effective in dynamically modulating corticospinal interaction accordingly.

Next, we investigated corticospinal coherence. Most previous studies using weak to medium force output have reported coherence primarily in the beta band, between 15 and $25 \mathrm{~Hz}(12-15)$. Coherence at frequencies in the gamma band, between 30 and $80 \mathrm{~Hz}$, has so far been described during maximal voluntary contractions or during slow movements (15). In our paradigm, beta-band coherence was present both before and during stimulus presentation (Fig. 1A). During visual stimulation but in the absence of changes in motor output, gamma-band coherence was strongly enhanced (Fig. 1, A and $\mathrm{B} ; P<0.05$, nonparametric permutation test, corrected for multiple comparisons). The topography of beta-band coherence before stimulus onset was very similar to that of gamma-band coherence after stimulus onset (Fig. 1, C and D), which suggests that the same neuronal group or two spatially highly overlapping neuronal groups were involved. Obviously, subjects knew that they had to be ready to respond only when the stimulus was on. Beta-band coherence was present throughout the trial and was more pronounced before the onset of the visual stimulus; this finding suggests that it has a role in maintaining a steady-state force output, in agreement with earlier studies (12-15). In contrast, corticospinal gammaband coherence seemed to come into play when a movement signal was expected.

We thus compared time courses of the hazard rate and of corticospinal gamma-band coherence separately for the UP- and DOWNschedules. Corticospinal gamma-band coherence showed rapid dynamics. In the UP-schedule, it increased over time after stimulus onset (Fig. 2, E and F) and in close correspondence with the hazard rate $(r=0.87, P<0.0001$, nonparametric randomization test). In the DOWN-schedule, gamma-band coherence first increased rapidly after stimulus onset and then fell to baseline values (Fig. 3, E and F), again in close correspondence with the hazard rate $(r=0.86, P<0.0001)$. To test whether this effect is specific for the gamma band, we assessed the cross-correlation between the hazard rate and the time course of corticospinal coherence for all frequencies of the coherence spectrum. Indeed, a significant positive correlation was confined to frequencies between roughly 40 and $60 \mathrm{~Hz}$ for both the UP- and DOWN-schedules (Figs. 2G and 3G).

In addition to the long-range coherence of motor cortex with the spinal cord, local neuronal synchronization in motor cortex was also modulated by the task. Oscillatory motor cortical synchronization can be estimated from the power spectrum of MEG signals recorded over motor cortex. Motor cortical gamma-band power correlated positively with the hazard rate (UP: $r=0.54, P<0.0001$; DOWN: $r=$ $0.61, P<0.0001)$. In contrast, motor cortical beta-band power showed a negative correlation (UP: $r=-0.89, P<0.0001$; DOWN: $r=-0.62$, $P<0.0001)$. The time course of power and the frequencywise correlation between hazard rate and power are shown in Fig. 2, B to D, for the UP-schedule, and in Fig. 3, B to D, for the DOWN-schedule.

Our results show that readiness to respond and corticospinal gamma-band coherence are tightly coupled. Dynamic modulations of the readiness to respond induce corresponding dynamic modulations of corticospinal gammaband coherence and motor cortical gammaband synchronization. Because readiness to respond was rising in the UP-schedule but falling in the DOWN-schedule, the parallel changes in power and coherence cannot be merely an effect of elapsing time. Furthermore, because visual stimulation and motor output (21) were indistinguishable between a given trial in the UP-schedule and a given trial in the DOWNschedule, the observed effects must be ascribed to the implicitly learned hazard rates and thus constitute a purely cognitive modulation.

One concern is that the observed dynamics in corticospinal gamma-band coherence might be fully explained by the changes in local motor cortical gamma-band synchronization. Several earlier studies have found gamma-band power over different parts of the brain to predict shortened reaction times 
Fig. 3. (A to G) Effect of decreasing hazard rate on reaction times, cortical power, and corticospinal coherence. Format as in Fig. 2.

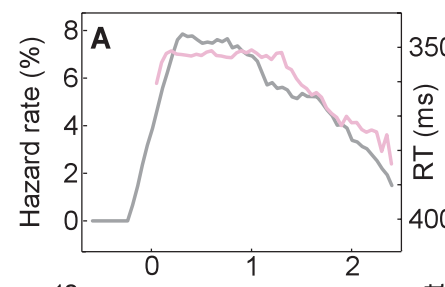

350 Hazard rate

(n) Reaction time

$\beta$-band power

$\gamma$-band power/coherence

Correlation coefficient
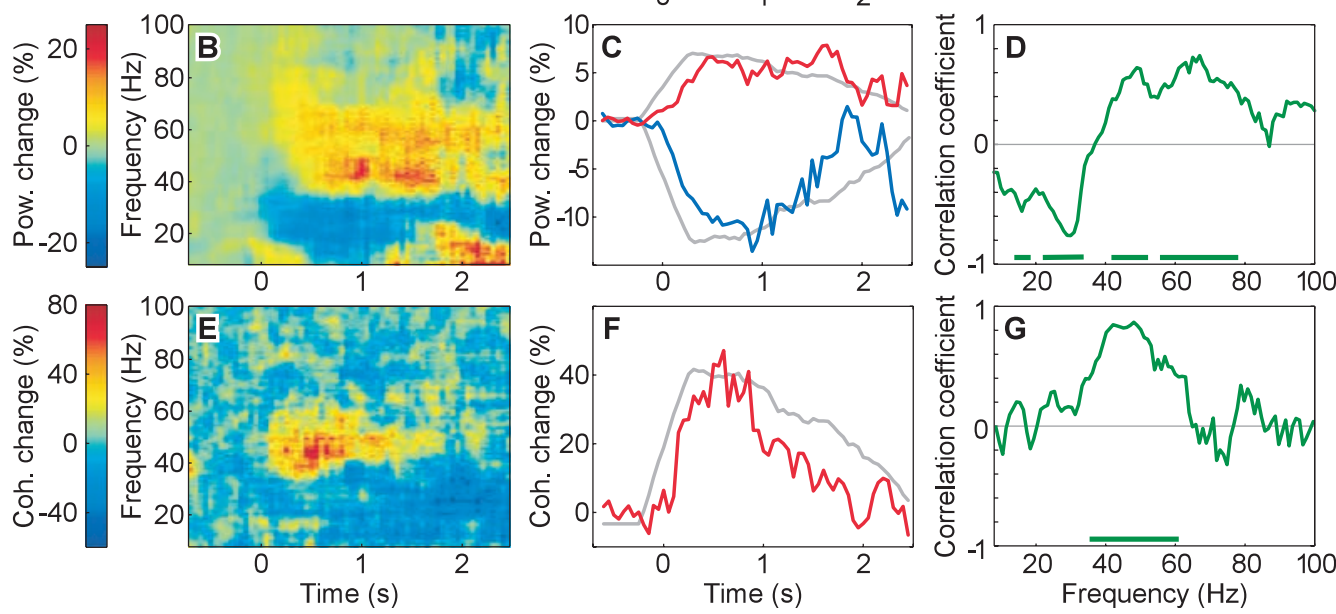

(22). The core of our hypothesis is, however, that coherence itself is a mechanism for effective corticospinal interaction, above and beyond local oscillatory neuronal synchronization. We therefore reanalyzed the dynamics of corticospinal coherence after eliminating trials until the average cortical power remained as constant as possible given the finite trial number. This left the magnitude and dynamics of gamma-band coherence essentially unaltered (20). Thus, the dynamics of corticospinal gamma-band coherence is not a mere consequence of the dynamics of local oscillatory synchronization, and the respective mechanisms appear at least partly independent. Previous studies have indeed revealed mechanisms that specifically modulate a neuron's tendency to phase-lock to rhythmic input (23) through a modulation of its ionic conductances.

Our results suggest a role for coherence in corticospinal interaction. Yet, in our paradigm, the corticospinal interaction was only one of the links in the chain from visual input to motor output. Coherence between different intervening areas might also contribute to the observed shortened reaction times during enhanced readiness to respond. Neuronal gamma-band coherence has been found in several other cases between distant neuronal groups along the visuomotor pathway (24-29) and has been implicated in visuomotor interactions.

We would like to speculate about the implications if neuronal coherence were a general mechanism of neuronal communication. Coherence likely renders neuronal interactions not only effective but also selective for the coherent groups. Input to a target group that is incoherent with the excitability fluctuations in this target group will be less effective. Often many neurons converge on a common target, but at a given moment, only part of the input is effective in influencing the target neuron's output. Selective coherence is an ideal candidate mechanism for the regulation of the efficiency of input. It would at the same time increase the impact of input that is coherent with the target and decrease the impact of input that is not coherent with the target. In addition, feedback from the target group of neurons would also be more effective at the coherent input than at the noncoherent input, even if it were anatomically directed to both. Our results suggest that neuronal coherence can serve neuronal communication and can be dynamically modulated by cognitive demands.

\section{References and Notes}

1. M. C. van Rossum, G. G. Turrigiano, S. B. Nelson, J. Neurosci. 22, 1956 (2002).

2. R. Azouz, C. M. Gray, Proc. Natl. Acad. Sci. U.S.A. 97, 8110 (2000).

3. E. Salinas, T. J. Sejnowski, J. Neurosci. 20, 6193 (2000).

4. R. Azouz, C. M. Gray, Neuron 37, 513 (2003).

5. H. Markram, M. Tsodyks, Nature 382, 807 (1996).

6. P. Fries, J. H. Reynolds, A. E. Rorie, R. Desimone, Science 291, 1560 (2001).

7. P. Fries, J. H. Schröder, P. R. Roelfsema, W. Singer, A. K. Engel, J. Neurosci. 22, 3739 (2002).

8. E. Salinas, T. J. Sejnowski, Nat. Rev. Neurosci. 2, 539 (2001).

9. P. H. Tiesinga, T. J. Sejnowski, Neural Comput. 16, 251 (2004).

10. D. J. Pinto, S. R. Jones, T. J. Kaper, N. Kopell, J. Comput. Neurosci. 15, 283 (2003).

11. M. Volgushev, M. Chistiakova, W. Singer, Neuroscience 83, 15 (1998).

12. J. M. Kilner, S. N. Baker, S. Salenius, R. Hari, R. N. Lemon, J. Neurosci. 20, 8838 (2000)

13. B. A. Conway et al., J. Physiol. 489, 917 (1995).

14. S. N. Baker, E. Olivier, R. N. Lemon, J. Physiol. 501, 225 (1997)

15. P. Brown, Prog. Neurobiol. 60, 97 (2000).

16. For conceptual clarity, we use the term corticospinal coherence instead of corticomuscular coherence. See
(20) for evidence that this coherence originates in motor cortex.

17. The hazard rate of an event at a certain time is defined as the conditional probability of the event occurring at that time, given that it has not yet occurred. See (20) for more information.

18. P. Trillenberg, R. Verleger, E. Wascher, B. Wauschkuhn, K. Wessel, Clin. Neurophysiol. 111, $1216(2000)$.

19. A. Riehle, S. Grün, M. Diesmann, A. Aertsen, Science 278, 1950 (1997).

20. See supporting data on Science Online.

21. To ensure that unavoidable small fluctuations in EMG activity did not confound the result, we used a stratification procedure that eliminated trials deviating from the mean EMG activity such that changes in EMG activity relative to baseline did not exceed 2\% (20). The described results were obtained with this stratification.

22. S. L. Gonzalez Andino, C. M. Michel, G. Thut, T. Landis, R. Grave de Peralta, Hum. Brain Mapp. 24, 50 (2005).

23. S. Schreiber, J. M. Fellous, P. Tiesinga, T. J. Sejnowski, J. Neurophysiol. 91, 194 (2003).

24. A. K. Engel, P. König, A. K. Kreiter, W. Singer, Science 252, 1177 (1991).

25. A. K. Engel, A. K. Kreiter, P. König, W. Singer, Proc. Natl. Acad. Sci. U.S.A. 88, 6048 (1991)

26. A. Frien, R. Eckhorn, R. Bauer, T. Woelbern, H. Kehr, Neuroreport 5, 2273 (1994).

27. M. S. Livingstone, J. Neurophysiol. 75, 2467 (1996).

28. P. König, A. K. Engel, W. Singer, Proc. Natl. Acad. Sci. U.S.A. 92, 290 (1995).

29. E. Rodriguez et al., Nature 397, 430 (1999).

30. M. R. Jarvis, P. P. Mitra, Neural Comput. 13, 717 (2001)

31. We thank H. Kleijnen and E. van den Boogert for technical assistance and R. Desimone, O. Jensen, C. Gielen, E. Maris, W. Singer, and I. Toni for helpful discussions and suggestions. Supported by Netherlands Organization for Scientific Research grants 452-03-344 and 051-02-050 (P.F.), Human Frontier Science Program Organization grant RGP0070/2003 (P.F.), and Danish Technical Research Council grant 26-01-0092 (R.O.).

\section{Supporting Online Material}

www.sciencemag.org/cgi/content/full/308/5718/111/ DC1

Materials and Methods

Figs. $\mathrm{S} 1$ to $\mathrm{S} 8$

References

2 November 2004; accepted 1 February 2005 $10.1126 /$ science. 1107027 